\title{
Airborne CCD camera pattern recognition analysis
}

\author{
Wei Han $^{a}$, Jinhe Bao ${ }^{b}$, Hongxiang Diao ${ }^{c}$, \\ Jun $\mathrm{Xie}^{\mathrm{d}}$,Xiuying Fan $^{\mathrm{e}}$ \\ Aviation University of Air Force,Changchun,China, 130022 \\ ahwa3@163.com, binhe_bao@sina.com, 'diaohongxiang@sina.com, ${ }^{d} x i e j u n @ 163 . c o m$, \\ efanxiuying@sina.com
}

\begin{abstract}
Keywords: Airborne CCD camera; enhance type of wavelet transform; adaptive filter; image recognition technology.

Abstract: CCD (charge coupled device) is one of the mainstream of image sensors, it plays a more and more important role in the real-time image processing and recognition. For airborne CCD image recognition problem, this paper proposes an enhanced method of wavelet transform. It can real-time processing of airborne CCD image, effectively filter out background noise, accurately locate and identify the important target. Compared with the traditional single pattern recognition methods, the method is fast and efficient target recognition, target positioning accuracy, advantages and the comparative test has been well validated, can be used as future airborne CCD image recognition technology development in a desirable direction.
\end{abstract}

\section{Introduction}

Airborne electric charge lotus root shut device CCD known as airborne CCD picture sensor often , may name the airborne picture controller too. Airborne CCD is a kind of semiconductor device, it can turn the optics image into the digital signal. The small photosensitive material planted into CCD is usually called picture element. It is many that pictures included on unit CCD are prime, the higher its resolution ratio of picture offered is. The function of CCD just like film, but distinguish that it is it that changes the optical signal into an electric charge signal. There are a lot of photodiodes with neat permutation on CCD, can respond to the light, and change the optical signal into an electric signal, amplifying and modulus change the circuit to change into a digital picture signal to sample by the outside.

CCD device as one of the present major picture sensors can be divided into line burst and kneaded dough a burst of two kinds, among them the line burst apply to more image scanner and Fax-machine, and CCD devices are in the high clear digital camera, consumption digital products and telemetering measurement of remote sensing, astronomy, such as digital camera ,etc. are measured, Aero-Space and goal are discerned,etc. the army and the people use the scientific research field used widelily. Can say so, CCD picture sensor's pattern process device as a kind of foundation, it can realize that information is obtained, conversion and expansion of the vision function, and can provide the ocular and true, visual picture information various and with abundant content of level, so has got widely application more and more in the modern society, there will be development in the future.

In recent years, burst of CCD play an more and more important role among the reconnaissance flight tasks the in common use type, it reconnoiters to the formation of image of the ground goal and interpretation nowadays become one of the effective means that the goal discern, but while the formation of image reconnoiters, often some factors exert an influence on the quality of the photograph, thus influence the interpretation result, these factors roughly include:

(1)Because of the change of the plane position and posture, it is out of shape to make the picture take place. 
(2)Influence of receiving the illumination and becoming the factor such as sensor that will be unavoidable in the course of formation of image, thus cause SNR to drop, the picture becomes fuzzy .

(3)In addition, the factors, such as atmosphere turbulance, observing light leak time of the goal and background noise gradient, etc. will influence the quality of the formation of image .

In order to solve this problem effectively, many kinds make an uproar method proposing and application. If traditional CCD picture goes to make an uproar the method can adopt the airspace and two major kinds of methods in the land strain waves to deal with frequently, as if the means of straining the wave, self-adaptation and straining waves etc. that the hitting value strains waves, neighborhood mean value. But in the course of practical operation, people find that the traditional method is while getting rid of the noise, will often make some detail information of the picture lose , cause the picture to be fuzzy and distorted. So, how can can not only get rid of the noise in the picture but also try one's best to protect the detail of the picture, one of the subject matters studied that it is the picture to go to make an uproar, is the main purpose of the method that this text proposes too.

As one of the main carriers of airborne pattern recognition, the airborne CCD camera discerns in current reconnaissance that the field is playing an more and more important role. These represent specific digital signal of information through treated follow-up, is it count mould vary analog signal of reducing into to undergo, resume the art of work as the information content included, is carried on the discernment of the goal by professional interpretation personnel finally, the concrete procedure block diagram is shown in Fig.1:

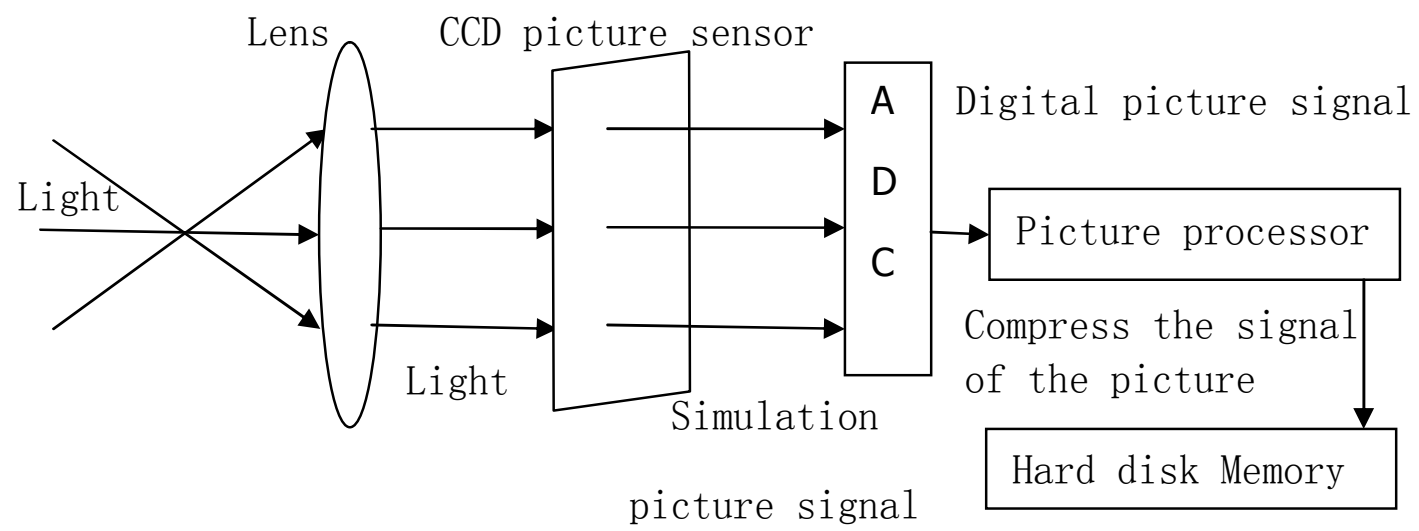

Fig.1 Airborne CCD camera workflow

\section{Images drawing and analysing}

The little wave of enhancement mode varies the idea of the method

While shooting and discerning the surface feature goal dynamically on the plane, in order to reduce the true colours which shoots the area better, a kind of pattern recognition method to usually adopt is that the small wave varies the law, it is to resolve the picture into sub pictures of different space, different frequency according to many resolution ratios that it carries on the basic thought analysed in pattern recognition. This kinds of method overcome effectively Fourier to vary one that exist shortcoming,and can to when land --Carry on the part and analyse, turn flexibly into and strain waves while drawing the some strange characteristic of signal and going on in the land frequently. Adopt light wave is it is it is it make an uproar signal, can strain effectively except that noise and keep the signal high-frequency message to include to deal with to vary, get the best recovery of the original signal. 
The small waves can all well get the result to vary, but consider that when environmental factor is comparatively the complicatedness, there are more noises in the possible picture, signal relatively dark a forgotten one, vary and reduce the picture with little wave only, will give a great discount in result. Where is such complicated situation correctly how more effective should? This text is on the basis of varying the theory in small unexpected turn of , the ones that combined the digital signal while dealing with strained the wave method in advance, the little wave of enhancement mode which has designed a kind of complex type varies the method.

The following of the formula:

$$
\begin{array}{ll}
\mathrm{C}_{\Psi}=\int_{\mathrm{R}} \frac{|\Psi(\omega)|}{|\omega|} \mathrm{d} \omega<\infty \quad \psi_{\mathrm{a}, \mathrm{b}}(\mathrm{t})=|\mathrm{a}|^{-1 / 2} \psi\left(\frac{\mathrm{t}-\mathrm{b}}{\mathrm{a}}\right), \mathrm{a}, \mathrm{b} \in \mathrm{R}, \mathrm{a} \neq 0 \\
\mathrm{~W}_{\mathrm{f}}(\mathrm{a}, \mathrm{b})=\left\langle\mathrm{f}, \Psi_{\mathrm{a}, \mathrm{b}}\right\rangle=|\mathrm{a}|^{-1 / 2} \int_{\mathrm{R}} \mathrm{f}(\mathrm{t}) \psi\left(\frac{\mathrm{t}-\mathrm{b}}{\mathrm{a}}\right) \mathrm{dt}
\end{array}
$$

The result of the picture is evaluated

The little wave of enhancement mode mentioned to this text varies the method, we have analysed the principle of this method and procedure of the concrete treatment picture from theory and algorithm at first. But the airborne picture shot of CCD camera, if deal with and discern with this kind of method, will get the result as scheduled, this needs further analysis and verification. In the course of real experiment, we have shot two groups of surface feature goal pictures with the airborne CCD camera separately, the strong noise of joining that people are in every group's picture , the direct photographs that so gets all seem very fuzzy, a bit more stray and a lot of. Then received after the small wave is varied and the little wave of enhancement mode is varied and dealt with separately the following result shown of picture of two groups of photos.

We are not difficult to find from the above two groups of experiments, after the small wave is varied and the little wave of enhancement mode is varied and dealt with parting by the picture of the noise pollution, the noise has all got certain elimination, but can be found from the further contrast of the picture : Vary and deal with the picture got and is closer to the primitive picture than the ones that only passed the small wave and varied and dealt with the picture got obviously through the little wave of enhancement mode, what the noise is dispelled is more abundant, what the part of detail of the picture is reduced too is more obvious. It is shown in Fig.2: 


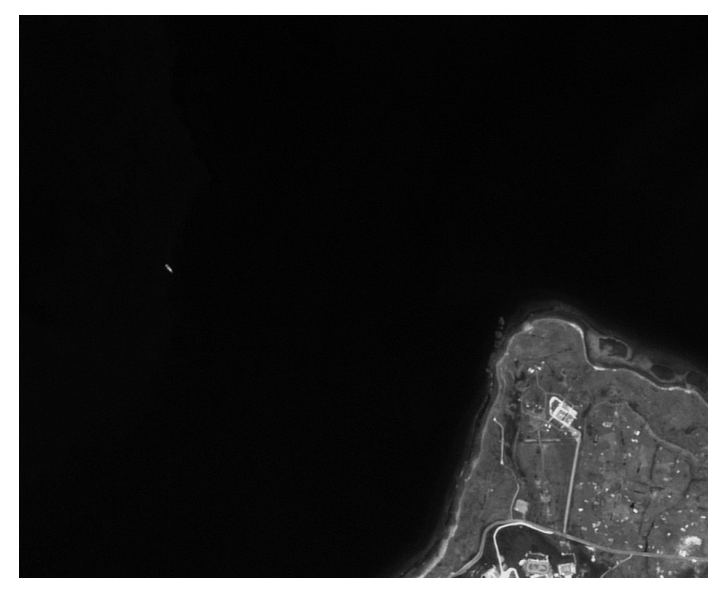

Primitive picture

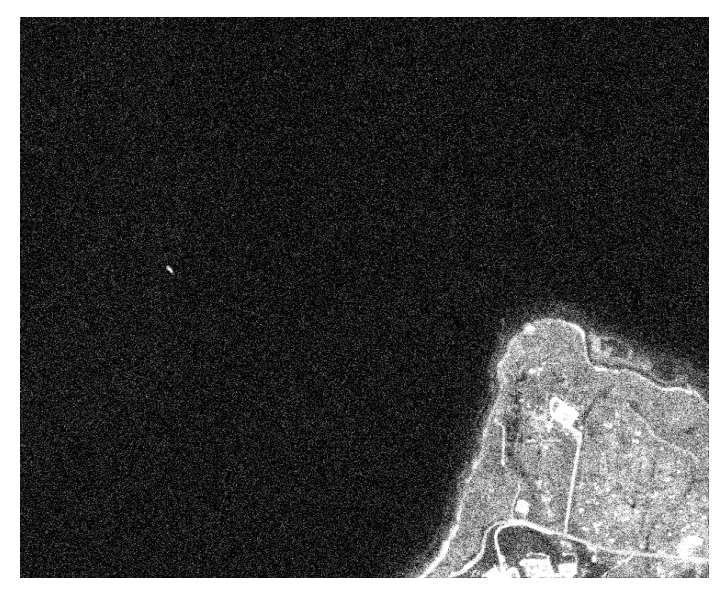

(c ) The little wave

varies picture

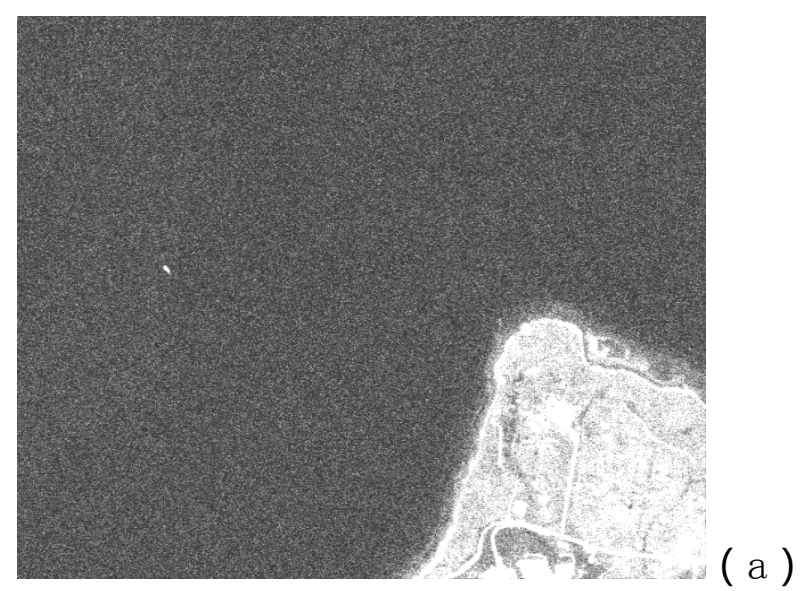

( b ) Noise picture

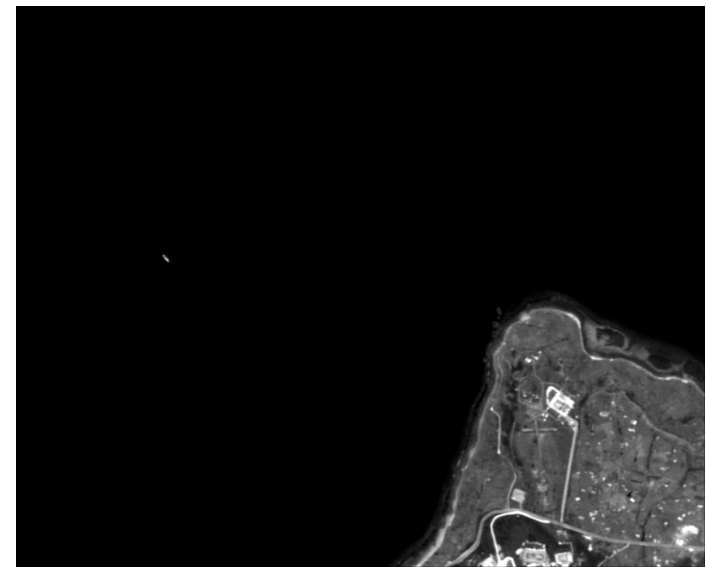

(d) The little wave of enhancement

mode varies picture

Fig.2 Result contrast of pattern process

\section{Conclusions}

One burst of CCD cameras will be unavoidable in the course of taking a photograph of the formation of image and is influenced by various kinds of factors the type in common use, these unfavourable factors are referred to as the noise, the influence of the noise will cause photos to become fuzzy, distorted, will become difficult to the discernment of the goal, in order to deal with this question effectively, this text has put forward a kind of small enhanced wave and varied the method in the tradition went to make an uproar on the foundation of the method. This kind of method varies the small wave the adaptive wave filter in theory and modern digital wave filter is conbined together organically, realization algorithm that the mathematics principle of the adaptive wave filter and small wave vary when and it should be systematic to introduce emphatically. In order to verify the feasibility of this method, this text airborne pieces of burst of CCD that camera shoot to is it make an uproar photo vary light wave method and enhancement mode little wave vary method deal with separately to include, the picture got and then carries on quality to evaluate according to the subjective quality evaluation criterion of the picture, and has carried on the statistics experiment. The little wave of enhancement mode of identification that the experimental result is very good varies the feasibility of the method, the effective method that this kind of method can be discerned as airborne CCD goal, and have very high value. 


\section{References}

[1] Jie Zhan, Xiaolei Jiang,etc..The little neural network of wave is used in CCD video array picture to make an uproar [J] The aircraft observes and controls the journal, 2010 (6). In Chinese

[2] Zhiyong Lei,Qunhua Liu,etc..The line is studied in one burst of CCD pattern process algorithms [D] Optics technology, 2002 (9). In Chinese

[3] Jianjun Jia.There are no man-machine big CCD camera remote sensing systems [J] Photoelectric project, 2006 (8). In Chinese

[4] Bo Li.Foreign aviation reconnoiters the development of the camera [J] Modern scientific instrument, 2013 (2). In Chinese

[5] Qunai Song,Qingyuan Huang.The application technology of CCD picture sensor and development trend,2005 (6). In Chinese

[6] $\mathrm{Yu} \mathrm{Hu}$,Jian Rong.Current situation of the development and prospect of CCD [J].Instrument and apparatus journal, 2005 (8). In Chinese

[7] Xiquan Gao, Yumei Ding.The digital signal deals with [M]. Electronic publishing house of University of Science and Technology of Xi'an, 2008 (8). In Chinese

[8] Hejin,Baoyu Zheng. Adaptive wave filter principle [M] Electronic Industry Press, 2010 (5). In Chinese 